\title{
MICROSCOPIC APPEARANCES OF CORNEAL GRAFTS
}

\author{
BY
}

\section{J. W. TUdoR THOMAQS}

CARDIFF

IN previous Papers (Trans. Ophthal. Soc. U.K., 1930 ; Proc. Roy. Soc. Med. Ophthal. Sect., June, 1930 ; Lancet (Hunterian Lecture), February 14, 1931, p. 335), a series of experiments on corneal grafting in rabbits was described and the results of various types of operation were given. Some of these grafts became infected, others became detached, others united. Some of those that united were opaque, some were nebulous, others, clear. In the papers referred to, the grafts were divided into six main groups according to the type of operation performed, as follows :-

1. Transplantation of the whole of the cornea with some conjunctiva.

2. Central or para-central grafts with stitches.

3. Central grafts without stitches.

4. Marginal grafts with stitches

5. Central grafts with parallel stitches passing over them.

6. Central grafts with "cross stitches."

Group 6 was divided into four sub-groups $(a),(b),(c)$ and $(d)$, according to the size of the graft and of the bed prepared for it. In the description that follows, the grafts will be referred to as belonging to one or other of the above groups.

All the grafts were of the whole thickness of the cornea. The changes in some of the infected grafts will first be described, and then the detached, and finally the united grafts. For convenience in reference, the number of the animal used in the experiment will be given thus, R.60=Rabbit No. 60 . Throughout the description the term "graft" will be used to indicate the transplanted corneal tissue, and the word "cornea" will indicate the rabbit's original corneal tissue as distinguished from the grafted tissue.

The microscopic appearances of transparent corneal grafts will be described separately in another paper.

\section{Infected Grafts}

Group 2. R.60. Killed seventh day. There is an invasion of the cornea by free leucocytes and epithelioid cells as well as an infiltration from an adherent iris. The graft itself, with the exception of a small portion in the centre, is infiltrated with leucocytes, 
small round cells, and epithelioid cells. These cells have entered from the anterior surface and from the cut ends, and tend to pass along the lines of the corneal fibres, pushing the lamellae apart.

Group 2. R.45. Killed eighth day. The graft is infiltrated with leucocytes, which have entered through the anterior surface and the cut ends, unaccompanied by blood-vessels.

Group 4. R.48. (See Fig. 1). This graft was nearly completely detached when the animal was killed at the seventh day. The

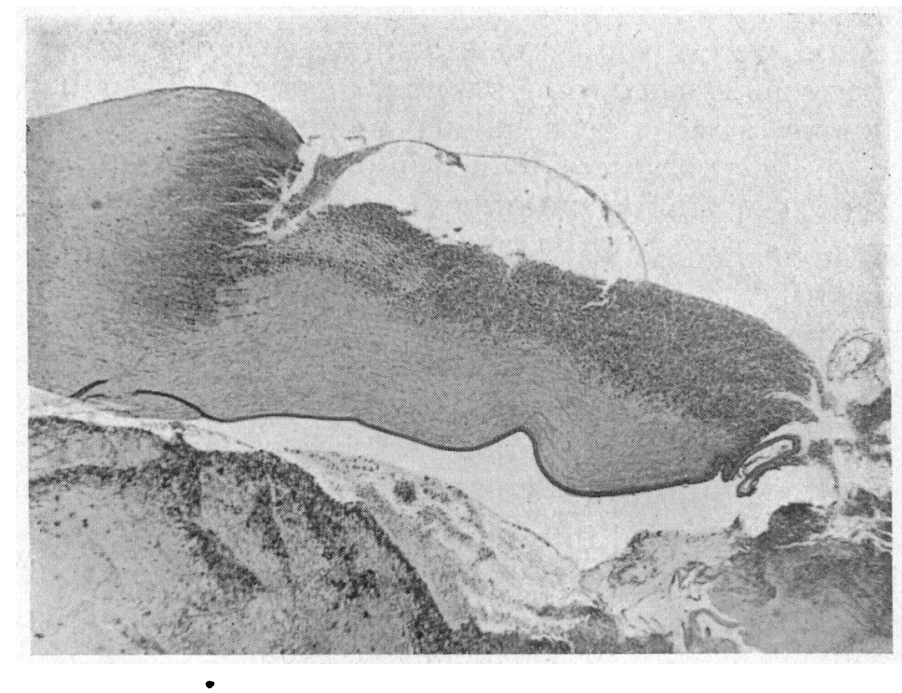

FIG. 1.

R. 48. Showing invasion of graft by cells from without. Magnification $\times 32$.

section shows that the graft has lost some superficial tissue and epithelium near the central margin, and that it has here been invaded by cells entering its anterior surface and at the cut margin. Thus the deep half of the graft is free from these cells, while the superficial half and the cut end have masses of ingrowing cells. These cells consist of leucocytes, or pus cells, and other irregular blue, round or oval cells, partly broken down, which suggests an epithelial origin. These cells have come from the surface and from without. These epithelioid cells are disintegrating and are soon almost unrecognisable.

Descemet's membrane is seen to be a definite barrier to the entry of these cells, so that the deeper layers of the graft are free from them. 


\section{Remarks on Infected Grafts}

The three infected grafts described show that the graft is apt to become infiltrated with cells from without. Some of these cells are of the nature of leucocytes or pus cells, which in all probability come from the iris or ciliary body and from the conjunctival sac; they penetrate the graft from the cut margins, and also from the anterior surface, while they are unable to penetrate through unbroken Descemet's membrane. They may be found in the graft in large numbers at the end of one week, and are not accompanied by blood-vessels.

Other cells, of an epithelial nature, also penetrate the surface and cut ends of the graft. These may be corneal epithelial cells, or other epithelial cells from the conjunctival sac. The cut ends of the cornea are liable also to be invaded by cells in a similar way, and if the iris is adherent to the cut corneal surface, leucocytes and young capillaries from the iris penetrate the corneal tissue as well.

\section{Detached or Partially Detached Grafts}

Eleven partly detached grafts were taken at various intervals ranging from four days up to one month after operation. One of these (R.46) was a marginal graft. All the others were more or less central in position. Three of these are illustrated and a short description of each follows.

Group 4. R.46. (See Figs. 2, 3 and 4). This graft was partly detached when the animal was killed on the thirteenth day after the

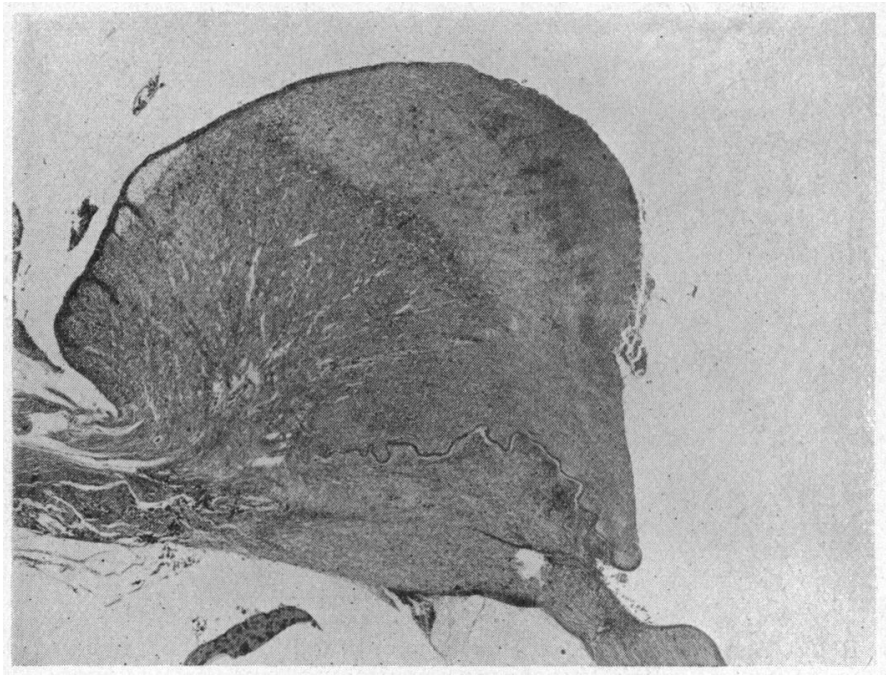

FIG. 2.

R. 46. Showing adherent iris, swollen graft, and invasion by blood vessels and leucocytes; new tissue deep to Descemet's membrane. Magnification $\times 13$. 


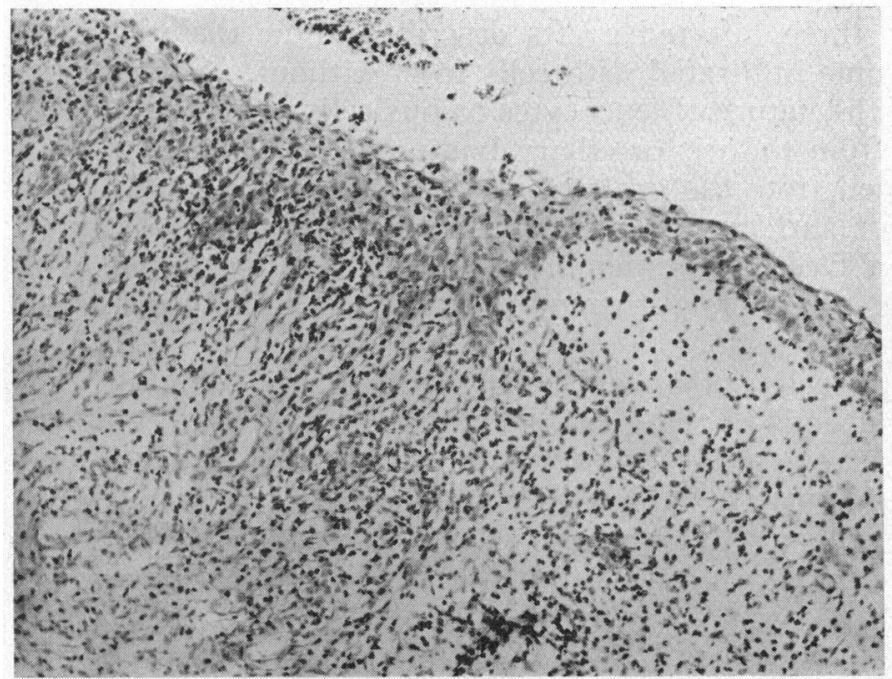

FIG. 3.

R. 46. Showing epithelium dipping into the graft. Magnification $\times 120$.

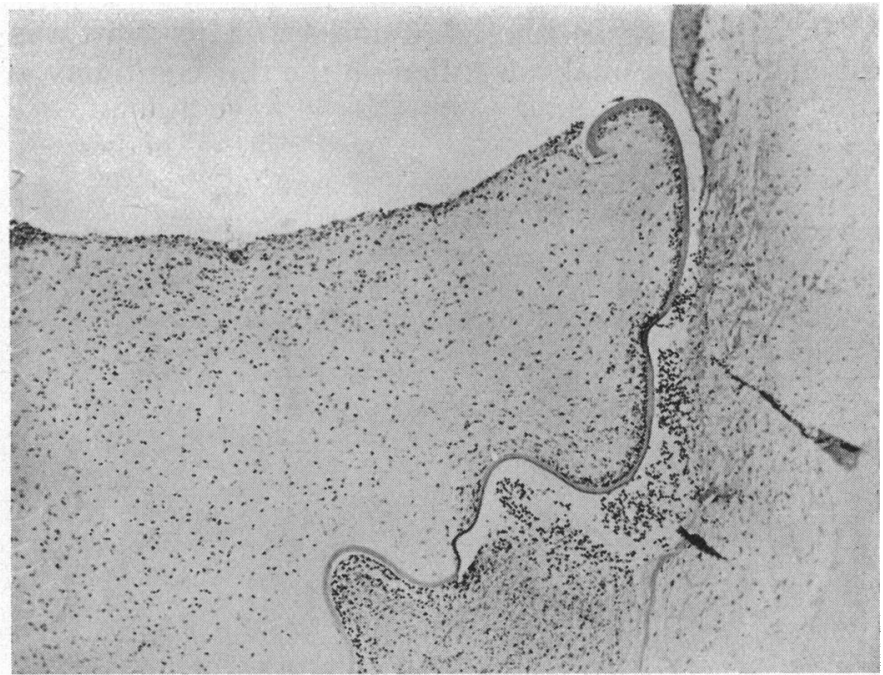

FIG. 4.

R 46. Showing how Descemet's membrane forms a barrier to the progress of cells ; leucocytes creeping in front of Descemet's membrane, and corneal epithelium growing down behind it. Magnification $\times 65$. 
operation. The iris is adherent to one end of the graft, and there is proliferation of the ciliary epithelium, which extends on to the adherent portion of iris. The other end of the graft lies anterior to the cut surface of the cornea, no union having taken place in this portion.

The graft itself is swollen, and three zones can be differentiated. The first zone is at the side to which the iris is adherent : this zone is densely nucleated and vascularised. Some of the cells have round, oval or spindle-shaped basophile nuclei, and these are more numerous than the leucocytes which are present. There are many newly formed capillaries, and the basophile cells appear to be derived from the walls of these capillaries. There is an intermediate zone which is much less densely nucleated; the nuclei are small and round, and the cells are mostly leucocytes.

The third zone is at the anterior surface of the graft, remote from the attached end. This zone is more densely nucleated than the intermediate zone, but much less than the first zone. Its nuclei are to a large extent broken up and consist of leucocytes, many of which exhibit eosinophilic characters, together with small round basophilic cells.

It appears that the superficial zone has become invaded by cells penetrating the anterior surface of the graft, while the deep zone is supplied with cells as a result of vascularization from the adherent iris.

The graft's Descemet's membrane is seen to act as a barrier to the passage of the mass of cells posterior to it, which are derived from the iris. A few leucocytes have found their way from without along the anterior surface of Descemet's membrane for a short distance. The graft epithelium is present over about half its surface, that is the portion adjacent to the attached margin, covering the first and the intermediate zones. It is absent over the superficial zone which has been described. The epithelium has grown over the cut end of the graft on to a small portion of the attached iris.

In places, the epithelial cells can be seen to pass into the substance of the graft in the form of plugs along the line of the corneal fibres. These cells seem to penetrate deeper into the graft in certain parts, and their large blue nuclei can be seen deep to the new and irregularly limited epithelium. The effect of this invasion by epithelial cells is that when viewed under a low power, the graft in this region is much more blue in colour than any other part of the graft or of the cornea. These cells appear to mingle with other cells derived from capillary walls in the substance of the graft.

Group 5. R.74. (See Fig. 5). This graft was partly vascularized but detached at one margin when the animal was killed one month 
after the operation. The graft is swollen and densely nucleated and its Descemet's membrane is thrown into folds. Newly formed blood-vessels are present in the superficial layers of the cornea, extending to the cut corneal surface; associated with these bloodvessels are numerous cells, which are round or oval, in the immediate vicinity of the blood-vessels, and more spindle-shaped in the less densely nucleated adjoining areas. Many of these cells appear to be derived from the capillary walls.

The iris is extensively adherent to the graft and has formed a considerable amount of new tissue behind the graft's Descemet's

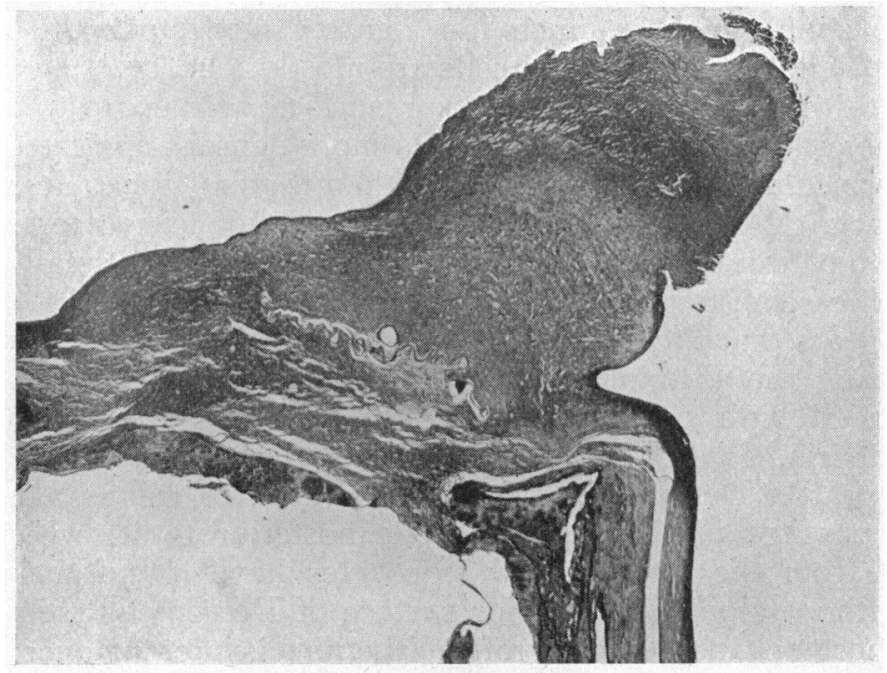

FIG. 5.

R 74. Showing protruding graft with its various zones; adherent iris; new tissue deep to Descemet's membrane; folds in Descemet's membrane. Magnification $\times 16$.

membrane. One end of the graft is separated from the cornea by a mass of leucocytes and some lighter stained oval cells ; this mass has an epithelial covering extending from the cornea to the graft for a short distance. The remainder of the graft has lost its epithelium for the most part.

The protruding superficial part of the graft is more or less amorphous and devoid of cells. The deeper tissues of the graft exhibit the following characteristics, described from without inwards and towards the attached end of the graft :-

(a) A zone containing broken-down leucocytes and nuclear débris. 
(b) A densely nucleated zone containing leucocytes without blood-vessels.

(c) A zone containing leucocytes, capillaries, and cells with large spindle-shaped nuclei.

It therefore appears that in this case there was little or no invasion of the graft by cells from without, and that there is a distinct difference in the character of the cells in various zones.

R.84. (See Fig. 6). This graft was slightly detached at one margin when the animal was killed on the sixth day after operation. At one end of the graft there are a few elongated endothelial cells between its Descemet's membrane and that of the cornea; the stroma of the superficial half of the graft is united to that of the cornea by exudate, which contains a few leucocytes. The other end of the graft is joined to the cornea by a larger mass of exudate containing endothelial cells, a few leucocytes, and other cells, possibly derived from the corneal fibre cells. This cellular invasion has extended into the graft for a short distance, and also into the cut surface of the cornea. The cut ends of the cornea, and the graft itself, are swollen. Leucocytes are present between the graft's posterior endothelium and Descemet's membrane.

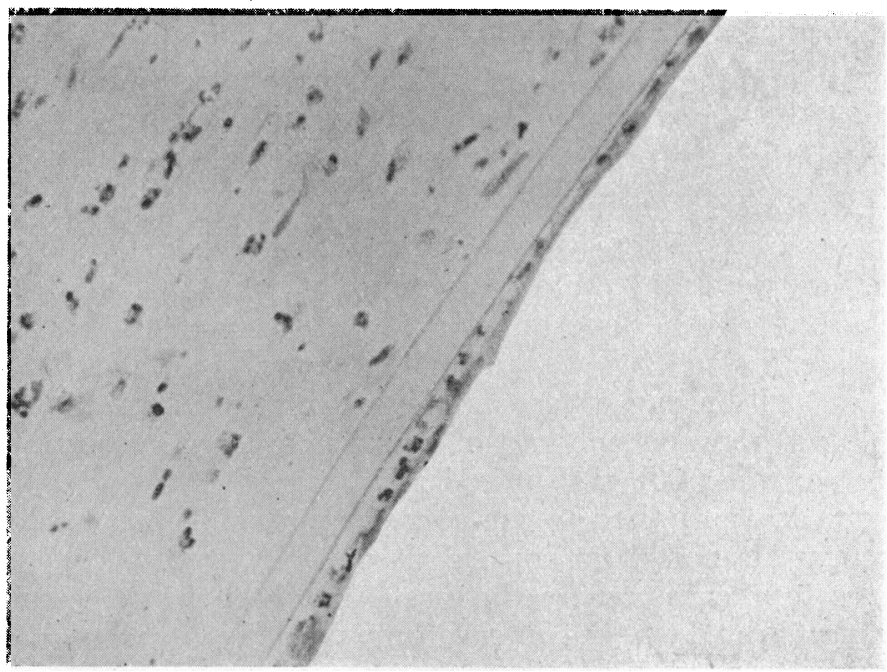

FIG. 6.

R. 84. Showing leucocytes between the endothelium of the graft and Descemet's membrane. Magnification $\times 530$. 


\section{Remarks on partly Detached Grafts}

(1) The Ten Central Grafts will first be considered :-

(a) Changes in the adjoining cornea.-Blood-vessels had extended from the periphery to the cut surface in four cases, the earliest being at eight days. Swelling and increase in thickness of the cut margins of the cornea were noticed in eight cases. In two of the early cases (four days and five days respectively), spindleshaped cells were found to have entered the cut ends of the cornea from masses of exudate adjoining them; this exudate had been formed mostly by the iris.

In two other cases (eighteen and twenty-one days respectively), blood-vessels from the adherent iris had found their way into the substance of the cornea through its cut margins. The corneal epithelium in all cases showed signs of proliferative activity.

In two cases (four days and five days respectively), the corneal epithelium had grown half-way down the cut margin of the cornea, and then on the surface of a mass of exudate intervening between the cornea and the graft.

In two other cases (six days and eight days respectively), the epithelium extended over the surface of a mass of exudate on to a portion of the surface of the graft.

In two other cases (nine days), the epithelium had grown half way down the cut corneal surface, and in one of these cases the epithelium passed on to a portion of the graft; one of these also exhibited growth of epithelium deep to the graft at one end, lying on the surface of some exudate.

The remaining four cases (fifteen, eighteen, twenty-one and twenty-eight days respectively), all exhibited a growth of the corneal epithelium on to the surface of the graft.

(b) Character of tissue intervening between graft and cornea.In the six cases where the animals were killed at or before nine days after operation, there was found in each case newly formed tissue at one or other end of the graft. In four cases this consisted of exudate formed by the iris and not yet vascularized. One of these, as well as one other case, exhibited vascularized iris tissue intervening between one end of the graft and the cornea. In one other case, the graft had united primarily at one end, and at the other end it was joined to the cornea by a small mass of exudate, apparently derived from corneal tissue.

In the four cases where the graft had been in place for fifteen to twenty-eight days, the intervening tissue was vascularized, and blood-vessels passed into the substance of the grafts.

(c) New tissue deep to the graft's Descemet's membrane.-In one case (four days) a film of exudate formed by the iris extended behind the graft. Four others (nine to twenty-eight days) exhibited 
new formation of vascular tissue behind Descemet's membrane, in the formation of which the iris had taken a large part.

(d) The graft's Descemet's membrane.-This membrane was found to act as a barrier through which stray cells in the anterior chamber could not passs. This was noticed particularly in the graft taken at eighteen days after operation.

Four grafts showed considerable wrinkling and folding of Descemet's membrane, and in one of these the membrane occupied a position folded over one end of the graft. In some cases, one end of the membrane occupied a position in front of the cut surface of the adjoining cornea.

(e) Changes in the epithelium of the graft.-A complete loss of epithelium over a considerable portion of the surface of the graft was noted in the first six cases (from four to nine days), and in two other cases (twenty-one and twenty-eight days), the epithelium was partly lost. Two of the grafts had epithelial coverings (fifteen and eighteen days). In one case (twenty-one days), the epithelial cells were found to have extended into the substance of the graft in certain parts. In two cases, the epithelium had grown so as to cover part of the cut end of the graft.

(f) The posterior endothelium of the graft.-In four cases, the endothelium was missing, one of these being the graft taken four days after operation.

(g) Swelling of the graft.-In eight out of the ten grafts now being described, there was considerable swelling of the graft, with marked increase in thickness.

(h) Changes in the stroma of the graft.-The nuclei in the graft were in a state of fragmentation in six grafts (four, five, six, eight, nine and twenty-eight days respectively). The graft was fairly free from cellular invasion in three cases (four, nine and nine days respectively). In two cases (eight and eighteen days respectively), there was an invasion of the graft by leucocytes and other cells, which had entered through the free end of the graft from outside. In four cases there was an invasion of the graft from the anterior surface by leucocytes and what appeared to be epithelial cells; this was noted as early as five days and as late as twenty-one days.

Six of the ten grafts showed invasion of the stroma by bloodvessels. The earliest was in the five-day graft, where a few capillaries, together with some spindle-shaped cells, had proceeded from the iris into a small portion of the attached end of the graft. The four grafts taken at four, six, nine, and nine days respectively, showed no such invasion by blood-vessels.

The eight-day graft was vascularized near its margin by bloodvessels supplied from the adherent iris, and by others which had 
grown in from the margins of the cornea. Of the remaining four grafts (fifteen and twenty-eight days), three were vascularized incompletely by vessels coming from the iris and from the margin of the cornea, and one was completely vascularized.

The appearance of the twenty-eight day graft has already been described, indicating the transitional characteristics in various zones extending from the deep vascularized portion to the superficial and much less cellular part.

(2) The Marginal Graft (thirteen days).

There was proliferation of the ciliary epithelium and invasion of the graft by blood-vessels. The corneal epithelium had grown on to some exudate, posterior to the graft. The cut ends of the cornea were swollen. Between the graft and the cornea there was vascularized tissue derived from the iris; this tissue also extended behind the graft's Descemet's membrane. Descemet's membrane was tortuous in outline and formed a barrier through which cells could not pass.

The graft's epithelium was partly lost; in places the epithelial cells passed into the substance of the graft. Some regeneration of epithelium was evident where it had covered a cut margin of the graft and passed on to the surface of iris tissue. The graft's endothelium was mostly lost, and the graft itself was swollen. The stroma of the graft showed fragmentation of its nuclei, and vascularization at the attached end.

\section{United Grafts}

Group 1. (Graft consisting of the whole of the cornea). R.3. This animal was killed nineteen months after the operation. At that time the centre of the cornea was roughened and opaque, while there was a partially clear narrow zone at the margin of the cornea. The anterior chamber was present.

The section shows thickening of the corneal epithelium, especially near the centre. The stroma contains blood-vessels and many nuclei, especially in the superficial half. At the partly clear marginal zone the corneal fibres are more regularly arranged, and the nuclei are thinner than in the opaque central portion; the epithelium also is less thick and more regular. Descemet's membrane is present and is rather thick $(0.022 \mathrm{~mm}$.). It possesses a single endothelial layer.

At the end of the graft's Descemet's membrane there is an adhesion of the iris to the graft. This adhesion is near the root of the iris, and from this point centrally the iris is free. Peripheral to the adhesion of the iris, there is a portion of the original Descemet's membrane extending to the angle of the anterior 
chamber. This piece of Descemet's membrane is quite as thick as the graft's Descemet's membrane and is $0.9 \mathrm{~mm}$. in length. It possesses an endothelial layer, and the iris is not adherent to its posterior surface. Probably this fact accounts for the absence of glaucoma in this eye, the aqueous could find its way past the peripheral anterior synechiae to the original angle of the anterior chamber to pass into the blood stream.

Group 2 R.16. This graft was opaque when the animal was killed three months after the operation.

The section shows the iris to be adherent to the graft at each end, and irregular fibres to have grown across deep to the graft. The graft's Descemet's membrane has been thrown into folds. The superficial fibres of the graft lie close together, but in the rest of the stroma there are irregular spaces between the fibres. The corneal fibre nuclei are present and stain well, but there are fewer nuclei in the centre of the graft than in the peripheral portion.

R.58. (See Fig. 7). This graft was opaque when the animal was killed eighteen months after the operation. The opacity was noted as being mostly in the deep portion of the graft.

The section shows the graft's Descemet's membrane wrinkled and in folds, while deep to it is a layer of vascularized tissue continuous with the iris tissue. The graft itself contains a few bloodvessels, some of which have come through the cornea and some

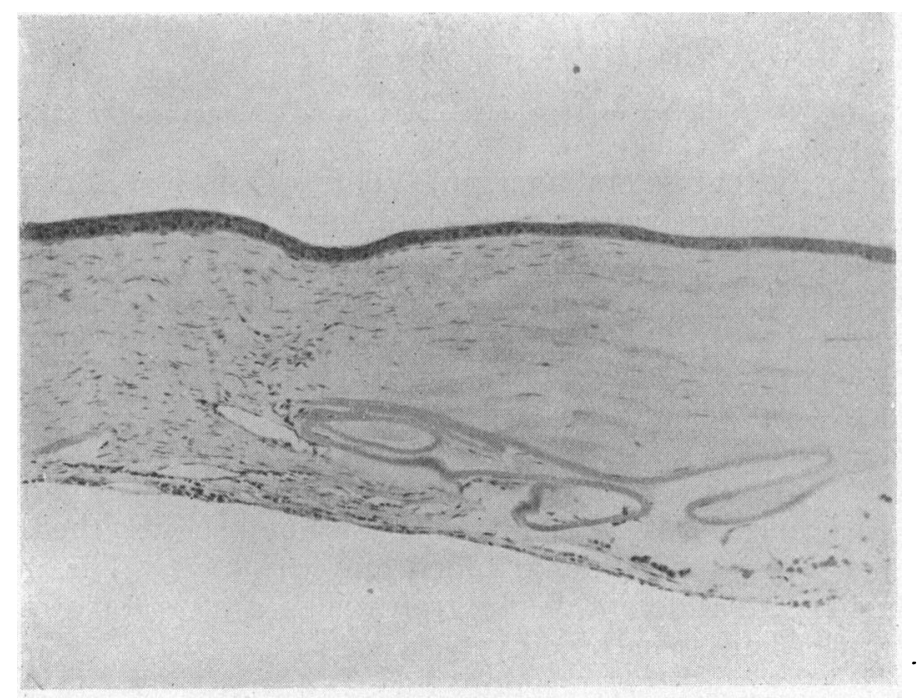

Fig. 7.

R.58. Showing vascular nature of new tissue deep to Descemet's membrane. Magnification $\times 117$. 
from the iris. The graft fibres are not separated by irregular spaces to any appreciable extent, and the nuclear content is not very high. The graft is covered with a layer of epithelium. The folds in Descemet's membrane, and the presence of vascular tissue on its posterior surface, account for the clinical fact that the opacity of the graft was mostly in its deep part.

Group 4. R.50. This animal was killed at the fifteenth day. The section shows considerable loss of the graft's epithelium, while the corneal epithelium has grown on to the surface of the graft near one margin. A few leucocytes have penetrated the surface and each end of the graft. There is a mass of tissue extending behind the graft's Descemet's membrane from the cut corneal

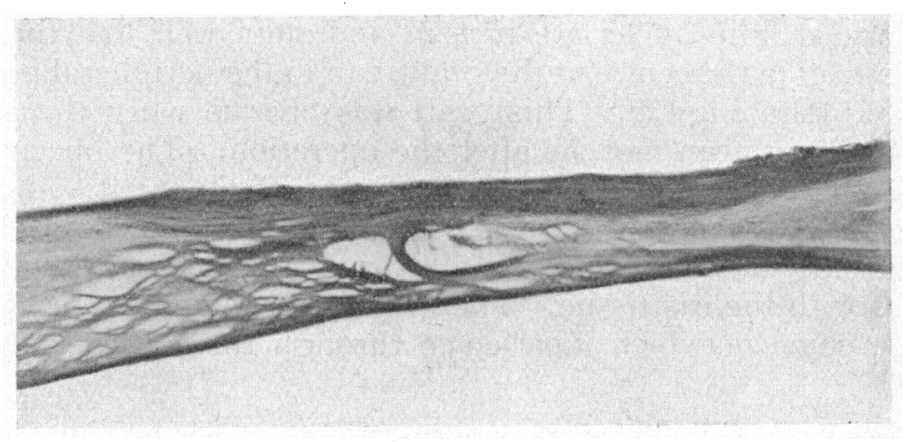

FIG. 8.

R. 55. Showing a new Descemet's membrane. Magnification $\times 55$.

margin to the cut scleral margin. This consists of fibro-blastic tissue, coming partly from the sclera, and largely from the iris ; it contains a fair amount of iris pigment, and is vascularized to some extent. This tissue extends forwards through a break in the middle of the graft's Descemet's membrane, and in this region a few leucocytes can be seen in the graft's stroma. The graft is thickened, and its anterior surface is raised above the general corneal surface.

R.62. This graft was opaque when the animal was killed ten months after the operation. The section shows the graft's Descemet's membrane much folded and wrinkled. The iris and lens capsule are adherent to the graft.' The graft stroma contains many nuclei and some blood-vessels.

Group 5. R.55. (See Fig. 8). This animal died sixteen months after the operation, the graft at that time being only slightly opaque and only slightly nebulous at the centre. The section shows an extensive new formation of Descemet's membrane 
joining the cut end of the corneal Descemet's membrane on one side to the posterior surface of the cut end of the corneal Descemet's membrane on the other side. The new membrane runs close to the posterior surface of the graft's Descemet's membrane, and in the central area the two membranes fuse together. No definite blood-vessels can be seen in the graft, and the iris is free.

Spindle-shaped spaces are present around the graft and also, to a lesser extent, in the superficial layers of the graft's stroma; the middle and deep thirds of the centre of the graft, however, exhibit a regular arrangement of the fibres and practically no spindle-shaped spaces.

The central area of the graft during life showed very little opacity, and the section shows this portion to have a regular Descemet's membrane without folds and closely adherent to the new membrane; the stroma shows only a comparatively small number of spindle-shaped spaces, and such as are present are found only in the superficial layers. Furthermore, it is to be noted that there is no new tissue formation behind the central portion of the graft's Descemet's membrane, apart from the new membrane which in this situation has fused with it.

Group $6(a)$. R.80. (See Fig. 9). This graft had a large area which was nearly clear when the animal died of old age ten months after the operation.

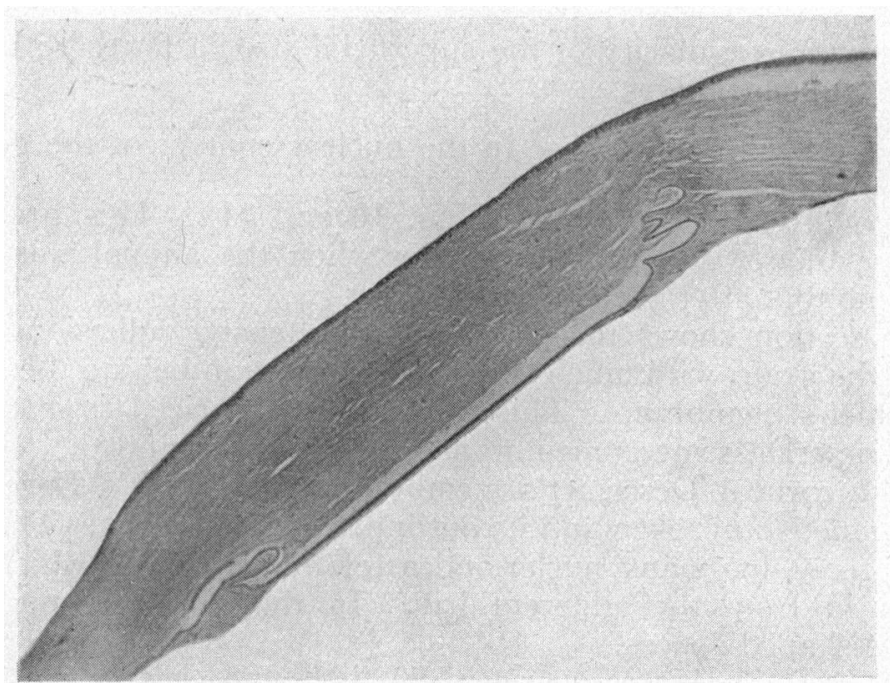

FIG. 9.

R. 80. A portion of the graft, showing a fold in the middle of its Descemet's membrane. Magnification $\times 34$. 
The section shows some folding of the graft's Descemet's membrane near the margins, and in this situation there is some new tissue deep to the membrane consisting of cells with long, pointed, straight nuclei, running more or less parallel to each other, behind which there is an endothelial lining. At the middle of the graft there is a fold in Descemet's membrane with a localized formation of new tissue similar to that described at the margins. Between this central fold and the margins, the graft's Descemet's membrane is regular and free from folds, and lined posteriorly by endothelium.

The graft stroma exhibits regular arrangement of its fibres for the most part, but in the superficial layers there are some spindleshaped spaces, and just deep to these the fibres are not clearly differentiated but tend to form an amorphous mass. The graft possesses an epithelial covering, the thickness of which varies in different parts, from one to seven cells thick. The iris is not adherent to the graft, although some pigment on the posterior surface of the corneal Descemet's membrane near the cut margin on one side indicates that there was an adhesion of the iris at this situation for a short time.

No blood-vessels can be seen in the graft. The area of graft which was nearly clear, shows the following microscopic appearances to account for the presence of slight opacity :-

(a) A fold in the middle of its Descemet's membrane with a localized new tissue formation behind it.

(b) Some irregularity of the superficial stroma fibres with some spindle-shaped spaces.

(c) A very slight increase in the nuclear content of the graft.

Group 6 (b) R.90. (See Figs. 10 and 11). This graft was quite opaque with an adherent iris when the animal was killed three months after the operation.

The section shows that there is an extensive adhesion of the iris to the graft, with much new tissue formation behind the graft's Descemet's membrane. This tissue is vascularized from the iris and a new Descemet's membrane has formed behind the tissue to join the corneal Descemet's membrane. The graft's Descemet's membrane is unbroken and its outline is slightly wavy. The graft stroma contains many nuclei and a few blood-vessels which have grown in from the adherent iris. In the graft are numerous spindle-shaped spaces.

The graft in this case was made the same size as the bed in the cornea prepared for its reception. The section, however, shows that the ends of the graft's Descemet's membrane are separated by a considerable interval from the cut ends of the corneal 


\section{Microscopic Appearances of Corneal Grafts}

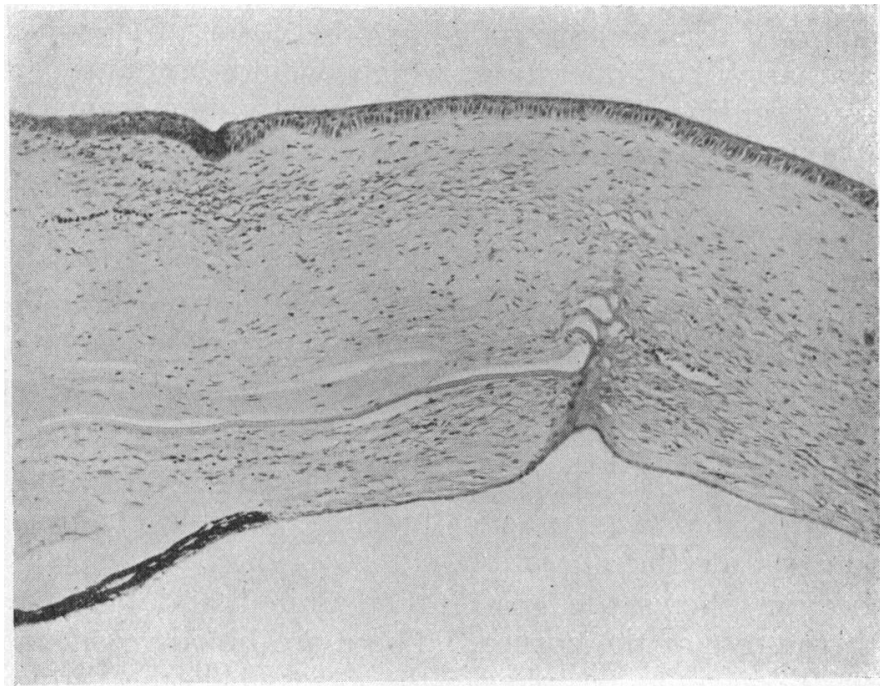

FIG. 10.

R. 90 Showing vascular nature of new tissue deep to Descemet's membrane; spindle-shaped spaces in graft, especially in the superficial half, which also shows an increase in the number of nuclei. Magnification $\times 83$.

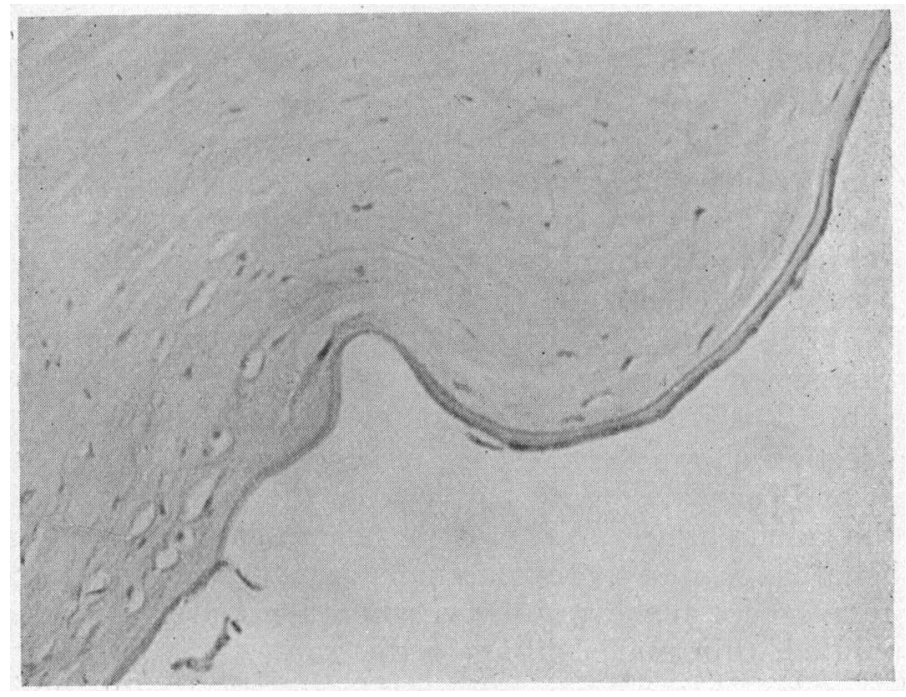

FIG. 11.

R. 90. Showing new Descemet's membrane where it is attached to the original corneal Descemet's membrane. Magnification $\times 201$. 
Descemet's membrane. In this connection, it is to be noted that the graft became protruded in the form of a cone about nine days after the operation, which would account for some waviness of outline of the graft's Descemet's membrane.

This waviness of outline necessarily implies a shortening of the linear distance between the two ends of the graft's Descemet's membrane.

This, coupled with the fact that the cut ends of the corneal Descemet's membrane were slightly curled up, accounts for the wide separation referred to between the graft's Descemet's membrane and that of the cornea. In the gap so formed, the iris was able to secure a good hold and became adherent.

Group $6(c)$. R.94. This graft was flat and well united, with a central area that was practically clear when the animal died twenty days after the operation.

The section shows the graft to be united and fairly well in line with the rest of the cornea. There are blood-vessels passing to the graft from the periphery; these vessels come through the cornea, and many capillaries are present at the junction of the graft and cornea. The centre of the graft has only a few small blood-vessels, and for the most part its fibres are regularly arranged without spindle-shaped spaces.

The nuclei in the graft are more numerous than normal, but the nucleation is much more marked at the periphery. The graft has a small adhesion of the iris at each margin. The iris has not apparently contributed much to the vascular supply of the graft. The interval between the graft's Descemet's membrane and that of the cornea is filled up by fibroblastic cells, which appear to have arisen partly from the cornea and partly from the iris. There is no new tissue formation behind the graft's Descemet's membrane except close to the margins. A good epithelial covering is present, as well as a single endothelial layer posteriorly.

R.93. This graft was opaque at the margins and nebulous at the centre when the animal died two-and-a-half months after the operation. The iris was adherent to the graft.

The section shows the presence of blood-vessels at the junction of graft and cornea, coming partly from the periphery and partly from the adherent iris. The graft has an epithelial and an endothelial layer, and there is no new tissue deep to its Descemet's membrane except at the periphery, where there are some long cells with pointed, thin nuclei filling in the gap.

Blood-vessels are absent in the centre of the graft; the stroma in this region has many more nuclei in the superficial layers than in the deeper layers. The fibres in the deep half of the graft are fairly regularly arranged. 


\section{Remarks on United Grafts}

The ten united grafts which have been described were taken at various intervals, ranging from fifteen days to nineteen months after operation. One of these was a graft consisting of the whole of the cornea together with some conjunctiva; two were marginal grafts; the remaining seven were more or less central in position.

(1) The Seven Central Grafts will be considered first :-

These may be divided into three groups, according to the degree of opacity present :-

(a) Opaque grafts.

(b) Grafts with a nebulous central area.

(c) Grafts with a central area nearly clear.

(a) Opaque grafts. - Three grafts were opaque (R.16, R.58, and R.90). Two of these (R.16 and R.90) were taken three months after the operation. In each case the iris was adherent and Descemet's membrane was thrown into folds. New tissue was present behind Descemet's membrane in each case, and in two of them this tissue was vascularized by the iris. In one case, there was a new Descemet's membrane, which was separated from the old membrane by some new tissue. Each of the grafts had bloodvessels passing to it from the cornea, supplemented in two cases by vessels from the adherent iris.

In two of the grafts there were irregular spaces between the fibres of the stroma. One of the grafts showed a close packing together of its superficial fibres. The nuclear content of each graft was increased, markedly so in two cases; the graft in which the nuclear content was not much increased had very few irregular spaces between its fibres, and the opacity in this case had been noted as being in the deep part of the graft. Epithelium was present in each of the three grafts.

(b)Grafts with a Nebulous Central Area.-There were two such grafts (R.55 and R.93), taken at sixteen months and two and a half months respectively after operation. The iris was adherent in one case. Descemet's membrane in each case was not in folds. New tissue formation deep to Descemet's membrane in each case was confined to the marginal part of the graft and was not present behind the central area.

One of the grafts appeared to be devoid of blood-vessels, and in the other graft they were only appreciable near the margins. The deep half of each graft showed a regular arrangement of its fibres, while the superficial third or so showed increase of nuclei with closely packed fibres in one case, and spindle-shaped spaces between the fibres in the other case. Each graft had an epithelial covering. 
(c) Grafts with a Central Area nearly clear.-There were two such graft's (R.80 and R.94) taken at ten months and twenty days respectively after operation. One of these had an adherent iris (R.94) and no folds in Descemet's membrane nor new tissue deep to Descemet's membrane, except at the margins. The other graft had one fold in the middle of its Descemet's membrane, and there was no new tissue formation behind this membrane except at the periphery.

The twenty-day graft was slightly vascularized at the margins, and in the other graft the blood-vessels which had been present had practically disappeared. The ten-month graft had spindleshaped spaces in the.superficial third of its stroma, while the other graft was free from such spaces. In each case, the graft contained more nuclei than normal, and each graft had an epithelial covering.

(2) The Two Marginal Grafts.

These two grafts were taken fifteen days (R.50) and ten months (R.62) respectively after operation, and each graft was opaque. In each case the iris was adherent and there were folds in Descemet's membrane, with new formation of vascularized tissue deep to the membrane. The ten-month graft had blood-vessels in its stroma, supplied from the scleral and conjunctival vessels, as well as from the adherent iris. The nuclear content of the tenmonth graft was decidedly increased, while the increase of nuclei. in the other graft was slight. There was much loss of epithelium in the fifteen-day graft.

(3) Graft Consisting of the Whole of the Cornea.

The graft described (R.3) was taken nineteen months after operation. It may be noted that the iris was adherent at the periphery, but that Descemet's membrane was not folded and had no new tissue deep to it; that the graft was vascularized, particularly in its superficial half, and that there were large, irregular spaces between its fibres. The superficial layers were closely packed together, with many nuclei, and the nuclear content of the graft was much increased. The graft possessed an epithelial covering, which was rather thick.

\section{General Relationship between the Degree of Opacity of the Grafts and the Microscopic Appearances}

The seven central grafts and the two marginal grafts will now be considered, and for this purpose will be divided into two Groups :-

(a) Seven grafts of two-and-a-half months and more;

(b) Two grafts of fifteen days and twenty days respectively. 
(a) Of these seven grafts, four were opaque, two had nebulous central areas, and one had a central area that was nearly clear. All these grafts had an epithelial covering. Each of the opaque grafts had an adherent iris, folds in Descemet's membrane throughout its extent, new tissue formation deep to Descemet's membrane (which in three cases was definitely vascular), and blood-vessels in the graft.

Of the two grafts with nebulous central areas, one had an adherent iris, neither had folds in Descemet's membrane, neither had new formation of tissue deep to Descemet's membrane except at the periphery, and the centre of each graft was practically devoid of blood-vessels.

The graft with a central area that was nearly clear had no adhesion of the iris, and there was only one small fold in the middle of the Descemet's membrane, with a localized small mass of avascular new tissue at this spot. The few blood-vessels which had been present in the graft had largely disappeared.

Increase in the number of nuclei in the graft was present in each of the four opaque grafts, and in three of them the increase was considerable. A definite increase of nuclei was present in one of the nebulous grafts, while the graft with an area that was nearly clear showed only a slight increase.

Irregular spaces between the graft's fibres were present in three of the opaque grafts, in one of the nebulous grafts, and in the graft with an area nearly clear. In the last two, however, these spaces were only found in the superficial third of the graft.

One of the opaque and one of the nebulous grafts showed a new Descemet's membrane throughout its whole extent. In the former, the new membrane was separated from the old membrane by new tissue, while in the latter the two membranes were fused together in the central area of the graft.

(b) Of these two grafts, one had a central area that was nearly clear, and one was opaque. Each had an adherent iris. The opaque graft had folds in Descemet's membrane, and vascularized new tissue deep to Descemet's membrane, whereas the other graft had no folds in Descemet's membrane, and new tissue formation deep to that membrane was confined to the margins.

There was an increase in the number of nuclei in each graft, but the grafts were not yet vascularized. The opaque graft showed much loss of epithelium.

\section{Degree of Swelling of the Grafts}

The following observations on the swelling of the graft are of interest in relation to the age of the graft and the depth of opacity present. 
Measurements of the graft were made from the surface down to Descemet's membrane and did not include any new tissue deep to Descemet's membrane. Increase or decrease of thickness of the grafts was estimated and is here given in terms of percentage increase or decrease from the average thickness of the cornea in the same eye. :-

(a) Two grafts taken at fifteen days and twenty days respectively. The graft with an area partly clear showed an increase in thickness of 15 per cent., while the opaque graft showed 140 per cent. increase.

(b) Three grafts taken at two-and-a-half to three months.

The graft with a nebulous centre showed an increase in thickness of 30 per cent., while the two opaque grafts showed 153 per cent. and 58 per cent. increase respectively-an average of 105 per cent.

(c) Four grafts taken at ten to eighteen months.

One nebulous graft showed a decrease in thickness of 66 per cent., and one graft with a partly clear area was the same thickness as the cornea. Two opaque grafts showed increase of thickness of 36 per cent. and 172 per cent. respectively, an average of 104 per cent.

From the above details, it will be seen that a certain amount of swelling of a corneal graft is present at two to three weeks after the operation, and the swelling is more marked in an opaque graft; that these grafts (none of which was perfectly clear) were all increased in thickness (with one exception) at periods varying from two-and-a-half to eighteen months, and that this increase was, on an average, more than 100 per cent. in the case of the completely opaque grafts, but not more than 30 per cent. in the grafts which were not completely opaque. One of the nebulous grafts showed a considerable decrease in thickness at sixteen months.

\section{Conclusions}

From the details enumerated in this paper, it will be seen that there is a considerable difference between the microscopic appearances of infected or partly detached corneal grafts and well united grafts. The features of each type have been given and summarised. There are also differences between the microscopic appearances of completely opaque grafts and those of grafts which are partly clear.

Dense opacity of a corneal graft appears to be associated particularly with the following features :- 
(a) Adhesion of the iris to the graft.

(b) Folding of Descemet's membrane.

(c) New tissue formation behind Descemet's membrane (often vascularized).

(d) Vascularization of the graft from the periphery through the cornea.

(e) Vascularization of the graft from an adherent iris.

(f) Increased nucleation of the superficial layers of the graft, associated with previous superficial vascularization.

(g) Large increase in the nuclear content of the graft.

(h) The formation of irregular spaces (sometimes spindle-shaped) between the graft fibres, thus causing irregular refraction and diffraction of light.

(i) Relatively large increase in thickness of the graft.

Conversely, the less these factors are present, the more likely is the graft to be clear.

The above conclusions are in accord with the results of the experiments as published (Lancet, February 14, 1931). Thus it was noted that an adhesion of the iris to the graft prejudices the result in regard to transparency. It has now been shown that such an adhesion of the iris tends to produce at least four of the features enumerated above, viz.- $(a),(c),(e)$, and $(g)$.

It can also be understood how the formation of a gap at one side of the graft (cf. partly detached grafts) tends to produce invasion of the graft by cells from without, as well as vascularization of the graft proceeding from the attached end, with consequent impairment of clarity of the graft. Furthermore, the iris is apt to become adherent where there is even a slight partial detachment of the graft, and blood-vessels are very likely to grow in from the iris as well as from the periphery of the cornea.

The swelling of the graft and of the cut margins of the cornea in the early days after operation, which was noted during the performance of the experiments, is confirmed by the microscopic features which have been described. It is worthy of note that this fact has an important bearing on the whole problem of corneal transplantation and was one of the underlying principles accepted in formulating the operative procedure which gave the most successful results. 